Original Article

\title{
Enhancement of motor skill learning by a combination of ideal model-observation and self-observation
}

\author{
Hitomi Nishizawa, RPT, MS'1), Teiji Kimura, RPT, PhD ${ }^{1 *}$ \\ 1) School of Health Sciences, Faculty of Medicine, Shinshu University: 3-1-1 Nagano, Asahi, \\ Matsumoto 390-8621, Japan
}

\begin{abstract}
Purpose] In sports physical therapy, video of a patient's movement or of a skilled model's movement has been used as observational learning methods for injury prevention and movement modification. Positive effects of model video observation have been reported. This study aimed to clarify the effect on motor skill learning using a combination of model-observation and self-observation, which is thought to act as an enhanced method for active error detection by comparing model-observation and self-observation alone for acquisition of correct sports movement. [Subjects and Methods] Forty-five healthy females were randomly allocated into three groups comprising model- and self-observation, model-observation, and self-observation. The motor task performed was a half golf swing using an elastic club. Shoulder grip angle between both shoulder lines and the acromia grip strength were measured as an index of body rotation using a three-dimensional motion analyzer. Change in the shoulder grip angle in the three groups was analyzed at pre-, immediate delayed retention, and delayed retention tests. [Results] A significant difference in shoulder grip angle was observed among the three groups for the immediate delayed retention test. The combined model and self-observation group had a value closer to 90 degrees compared to the other two groups. [Conclusion] Observation combining model and self-observation exerted a positive effect on short-term motor skill learning.

Key words: Action observation, Active error detection, Motor learning
\end{abstract}

(This article was submitted May 12, 2017, and was accepted Jun. 5, 2017)

\section{INTRODUCTION}

Sports physical therapy is a specialized subset of physical therapy practice that focuses on health care management of physically active individuals that have been injured in or aspires to return to athletic endeavors. A sports physical therapist establishes a customized plan of injury prevention, injury management, or performance enhancement in order to enable and maximize the athlete's participation in sports activities ${ }^{1,2)}$. In sports physical therapy, secondary prevention of overuse or misuse disorders, such as tennis elbow, thrower's elbow, and golfers elbow become important tasks ${ }^{3-5}$. For the secondary prevention of such sports disorders, reduction of pain, increase of flexibility and muscle strength, and modification of incorrect sports movements are required in physical therapy treatment. Modification of incorrect sports movements is an especially important task for secondary prevention of sports disorders, by eliminating misuse in actual sports activities after a comeback to the sports fields. For the modification of incorrect sports movements such as a greater elbow carrying angle ${ }^{6)}$ in golfers elbow ${ }^{7}$, verbal instruction, demonstration by a therapist, video observation of correct sports motion, and knowledge of results (KR) as feedback has been used as intervention methods. To facilitate the modification of an incorrect movement, active error detection by the learner needs to be part of the cognitive process. Observational learning is a known method to

*Corresponding author. Teiji Kimura (E-mail: tkimura@shinshu-u.ac.jp)

(C2017 The Society of Physical Therapy Science. Published by IPEC Inc.

(c) (1) $($ This is an open-access article distributed under the terms of the Creative Commons Attribution Non-Commercial No Derivatives CC. ${ }_{\text {BY }}$ ND (by-nc-nd) License. (CC-BY-NC-ND 4.0: https://creativecommons.org/licenses/by-nc-nd/4.0/) 
understand the correct movement in order to prevent such sports disorders. Generally, in sports physical therapy, therapists select motor tasks based on the characteristics of the sports disorders of the patients. Motor tasks are performed after verbal instruction and demonstration by the therapist. Then, the patient performs the motor task as instructed, and the difference between the instructed task and the movements actually performed by the patient is obtained from the therapist as $\mathrm{KR}^{8-10)}$. Thereafter, the patient is required to modify their movements for the next practice based on a comparison between intrinsic somatosensory feedback and KR. These processes for performing motor tasks have been carried out for motor learning in the field of sports physical therapy.

In this motor learning process, the method of instruction used by the therapist is important for effective motor learning and for aiding in easy understanding of a motor task, active error detection, and active error reduction by the patient. The manner of active error detection based on observational learning has been classified into three types based on previous studies: model-observation $(\mathrm{MO})^{11)}$, where the patient observes the ideal model movement; self-observation $(\mathrm{SO})^{12)}$, where the patient observes their own movement; and the model and self-observation (MSO), which is a combination of MO and SO. MO has also been defined as action observation ${ }^{13}{ }^{14}$, where an observer can understand what other people are doing by simulating the actions and the outcomes that are likely to follow from the observed motor act ${ }^{13}, 15,16$ ). In particular, the method of referring to another person's image has been regarded as MO. The positive effect of MO, achieved by observing the movement of another expert model via video, has been reported in motor learning for rhythmic gymnastics athletes ${ }^{17}$. On the other hand, $\mathrm{SO}$ is defined as observing the adaptive movement of one's self ${ }^{18)}$. Moreover, regarding the effect of SO, Lefebvre et al. ${ }^{19)}$ and Farquharson et al. ${ }^{20)}$ suggested that using video feedback significantly improved subjects' skills. In this way, since MO can obtain the correct motor images regarding the target motor task ${ }^{17)}$ and SO causes self-awareness about errors in a person's performance, these $\mathrm{SO}$ are regarded as information processing that correlates with active error detection $^{21)}$. From these previous studies, we hypothesized that MSO, which is combination of MO and SO, may be a more effective observation method for active error detection compared with MO or SO alone because we thought that MSO can lead to effective detection of any differences between the model's movement and learner's own movement by comparing the two kinds of apposed video information, without depending on memory. Regarding simultaneous observation via MSO, Barzouka et al. ${ }^{11)}$ investigated the difference in motor learning effect with respect to receiving a ball in healthy high school students, among three groups comprising combined $\mathrm{MO}$ and $\mathrm{SO}, \mathrm{MO}$ with verbal instruction, and no observation with verbal instruction. The context of verbal instruction between the MO group and the no-observation group were different. No significant differences were observed among the three groups regarding the outcome, and the verbal instruction component was believed to have affected the results. However, few studies have investigated the difference in motor skill learning between MSO, MO, and SO without verbal instruction.

Therefore, in this study, we decided to consider a more appropriate method of acquiring movement using observational learning with healthy subject as a fundamental study of prevention for sports disorders based on an incorrect sports movement. The purpose of this study was to clarify the difference of motor learning effects by comparing three different observational learning types, $\mathrm{MSO}, \mathrm{MO}$, and $\mathrm{SO}$, without verbal instruction for acquisition of correct sports movement.

\section{SUBJECTS AND METHODS}

Forty-five healthy females (mean age, 20.4 years; standard deviation [SD], 1.7 years) participated in this study. All of the subjects were novice in golf. All participants provided written consent prior to participating in this study, which was approved by the Shinshu University Institutional Review Board (Approval number 2665) and conformed to the Declaration of Helsinki.

The motor task was a continuous half swing motion (Fig. 1) using a specially made golf club for practice (SWING PRO II; MT Inc. Innovation, Japan). This motor task was selected because it was expected that a motor learning effect could be observed in this sport movement in novices, that there were moderate difficulties for healthy subjects, and also, it is a basic movement in golf. In addition, to make it easier for subjects to focus as narrowly as possible on observation points, a half golf swing instead of the full golf swing.

The club's shaft of SWING PRO II was made from elastic urethane resin (Fig. 2). This SWING PRO II was selected because a half golf swing, using this elastic urethane resin shaft, exaggerated the difference between the characteristics of beginners and a skilled model compared to a standard golf club in this preliminary study.

The half swing was performed in line with a rhythmical sound from an electronic metronome. All participants repeated half swings within 30 seconds without an actual golf ball. The model movement was demonstrated in a recorded video on an iPad (Apple Inc., CA, USA). The video was recorded by a teaching professional registered with the Professional Golfers' Association of Japan. In this video, the half swing was executed for 30 seconds using the SWING PRO II. This video was used as the model video for all groups.

MO, SO, and MSO were executed by observing the video using an inexpensive editorial iPad application (Fun! Lesson, ISAC Inc., CA, USA), which could simultaneously display the videos of MO and SO alone and MSO (Fig. 3A-C).

During a pre-test, all participants performed repeated half swings for 30 seconds using an electronic metronome at $62 \mathrm{bpm}$ after observing the video of the model movement for 30 seconds. The metronom's beat ( $62 \mathrm{bpm}$ ) was determined based on the actual model's beat as the optimal rhythm of the half swing. Thereafter, the 45 participants were randomly allocated to 


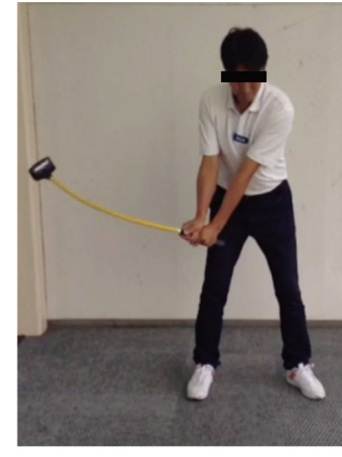

A

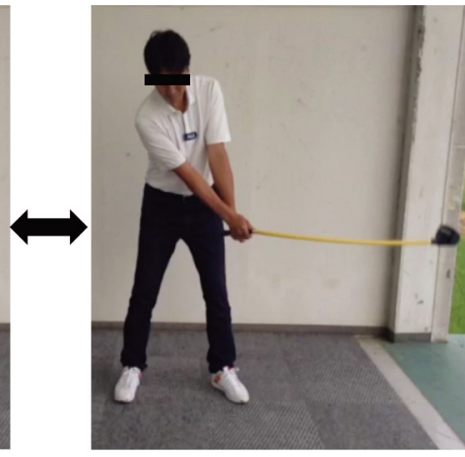

B
Fig. 1. Method of the continuous half swing

Half swing refers to an action of swinging the head of a golf club from the position of the right waist to the position of the left waist. In this study, subjects executed this half swing continuously to the sound of a metronome. A indicates take-back and B indicates the follow-through.

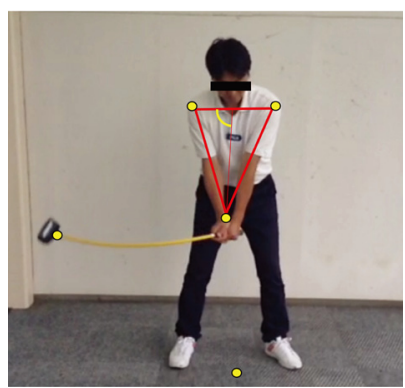

Fig. 2. Marker position and angle $\alpha$

This photograph shows the half swing in golf as recommended by the Professional Golfers' Association of Japan. The golf club was made from elastic urethane resin. Five markers were indicated as yellow circles. The angle of the take-back side between the shoulder line which connected both acromia and the line which connected the midpoint of the shoulder line and grip marker attached to the right radial styloid process were defined as $\alpha$ and is indicated by the yellow curve.

three groups comprising 15 participants each, and the motor task was executed in the acquisition phase 5 times a day for 5 consecutive days as follows: (1) the video of the model movement was observed for 30 seconds, (2) the half swing was practiced for 30 seconds, and (3) only the model video was observed (MO), the participant's own half swing video was observed (SO), or the model video and the participant's own half swing video was observed (MSO) (Fig. 4).

An immediate delayed retention test was performed after the final practice on the final day of the acquisition phase and a delayed retention test was performed one week after the immediate delayed retention test. The procedures for the immediate delayed retention test and delayed retention test were identical with that of the pre-test.

Five markers were affixed to both acromia, the right

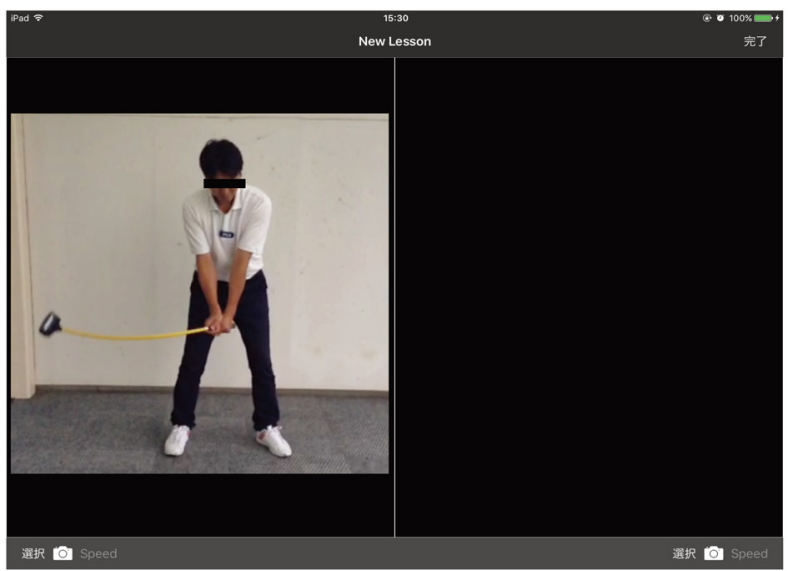

3-A.

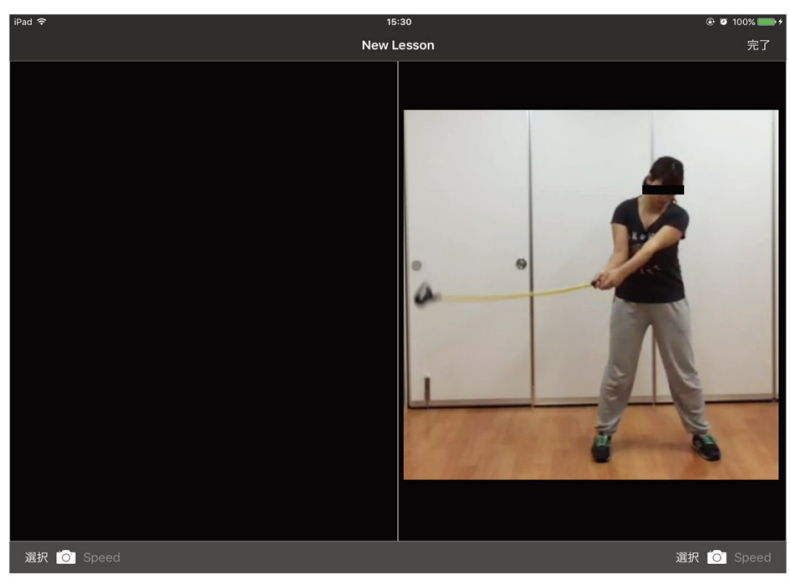

3-B.

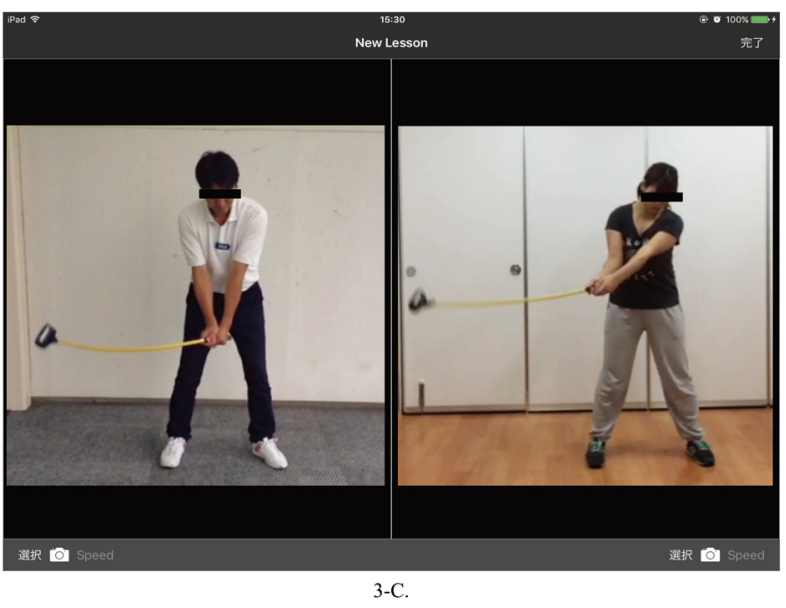

Fig. 3. View of iPad application "Fun! Lesson"

Subjects observed the movement using the application called "Fun! Lesson" on the iPad. A: This screen was observed by the subjects of the MO group and was the image when observing the video of model movement. B: This screen was observed by the subjects of SO group and was the image when observing the video of self-movement. C: This screen was observed by the subjects of the MSO group and was the image when observing the video of model and self-movement.

MO: model-observation; SO: self-observation; MSO: model and self-observation 


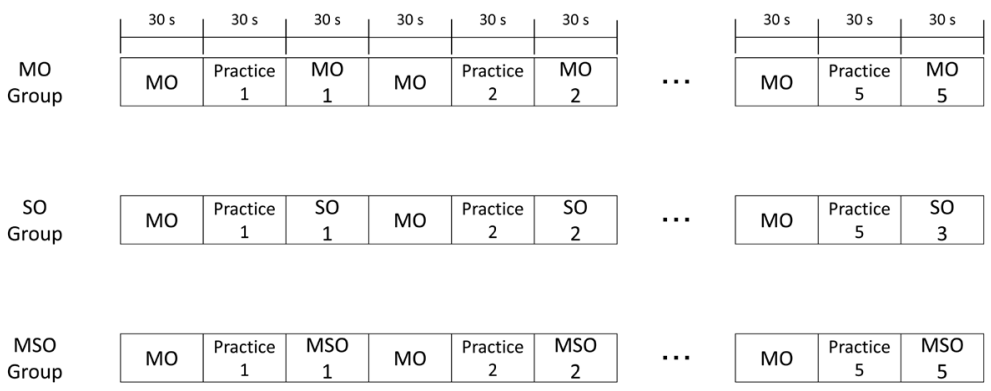

Fig. 4. Experimental sequence

All subjects were allocated into the three groups randomly. Then, the subjects observed the model image for 30 seconds. They practiced for 30 seconds to properly imitate the model movement. The MO group observed the model image, the SO group observed the video of self-practice images that was recorded at last-minute, and the MSO group observed the video of model and self-practice image at the same time. This was repeated 5 times a day, for a total of 5 days.

MO: model-observation; SO: self-observation; MSO: model and self-observation

Table 1. Comparison of the angle $\alpha$ of the three groups at the pre, immediate delayed retention, and delayed retention tests (Unit: degree)

\begin{tabular}{lccc}
\hline & Pre-test & Immediate delayed retention test & Delayed retention test \\
\hline MO & $82.3 \pm 2.9$ & $82.7 \pm 1.9$ & $83.4 \pm 5.3$ \\
SO & $81.0 \pm 5.4$ & $78.7 \pm 3.9$ \\
MSO & $83.8 \pm 4.7$ & $84.6 \pm 7.8$ & $80.2 \pm 5.4$ \\
\hline
\end{tabular}

$($ Mean \pm SD), MO: model-observation; SO: self-observation; MSO: model and selfobservation, ${ }^{*} \mathrm{p}<0.05$

radial styloid process, the head of a SWING PRO II golf club, and the floor to indicate the lowest position of the swing trajectory. The participants' half swing performances were measured using a three-dimensional analysis camera (3D camera; Opti Track V120 Trio, Natural Point Inc., OR, USA). The sampling frequency was $120 \mathrm{~Hz}$.

The motion analysis data were recorded onto a notebook PC and data analysis was performed using a biomedical signal analysis software (Kineanalyzer; Kissei Comtech Inc., Nagano, Japan).

Since the ideal half swing is outlined in a manual ${ }^{22}$ and shows that the triangular shape connecting both shoulders and grip should be maintained for correct body turn, the angle between the shoulder line that connects both the acromia and the line that connects the midpoint of shoulder and the grip marker attached to the right radial styloid process on the take-back side were analyzed as $\alpha$ in this study (Fig. 2). Therefore, if the body turn is correctly performed by maintaining the shape of the triangle made by the body and both upper limbs, the $\alpha$ value should be close to 90 degrees. If the correct body turn is not performed, and the triangle shape is not maintained, caused by asynchronous between trunk rotation and upper limb movement, the $\alpha$ value will be less than 90 degrees. Since the specificity of $\alpha$ was recognized at the preliminary study, $\alpha$ in the take-back side was set as the main index of correctness in the half swing in this study. Ten motions of middle data were analyzed during the 30 -second period.

Differences in $\alpha$ as an index of ideal skill for the half swing performance were compared using a two-way (group $\times$ time) repeated-measures analysis of variance (ANOVA) since a normal distribution was observed in all data of $\alpha$. The Tukey HSD test was performed for post hoc analysis. Statistical analysis was performed using the PASW Statistics software (version 24.0, SPSS, Inc., Chicago, IL, USA).

\section{RESULTS}

There was a significant main effect of group $(\mathrm{p}<0.05)$ and no significant interaction between group and time. A significant difference in $\alpha$ between the MSO and SO group was observed in the post-hoc test $(p<0.05)$ for the immediate delayed retention test; $\alpha$ in the MSO group had a value closer to 90 degrees compared to the SO group (Table 1). There were no significant differences in $\alpha$ in the inter-group comparisons in the pre-test and the delayed retention test. There were no significant differences in the intra-group comparison in all groups. 


\section{DISCUSSION}

The present study compared the effect of three different observation methods on short-term motor learning regarding the early stages of practicing the half swing with a golf club. The results revealed a significant difference in $\alpha$ between the MSO group and the SO group in the immediate delayed retention test, and that the MSO group had an $\alpha$ value that was closer to 90 degrees compared to the SO group. These results showed that the MSO group could preserve the triangular shape connecting both shoulders and grip for correct body turn during a series of half swings. Our findings suggest that when subjects learn a relatively difficult motor task through observation as in this study, using both model and self-videos has a more positive effect on short-term motor learning compared with learning via observing self-videos ( $\mathrm{SO}$ ) alone.

In the field of sports physical therapy for prevention of sports disorders, patients are generally asked to observe the therapist's demonstration as a model. Subsequently, the patient's performed movement is guided by verbal instruction or video information as KR from a therapist. To our knowledge, no study has investigated the effect of observing both the therapist's demonstration and the patient's own performed movement simultaneously in physical therapy. Our findings imply, with respect to the clinical practice of sports physical therapy, that the patient should observe both the therapist's demonstration and the patient's own performance simultaneously after each practice using inexpensive editorial applications to improve short-term motor learning. Although no previous study has reported the positive effect of MSO, our findings suggests that MSO is more effective for active error detection via the simultaneous observation of both the model task performance and the learner's own movement compared with SO alone just after the practice period as mentioned by the immediate delayed retention test.

The results for the delayed retention test indicate that the maintenance of motor learning effect revealed no significant differences among three groups. Furthermore, no significant intra-group differences were observed in the three groups.

Although in previous studies, regarding motor learning, authors often performed the delayed retention test 24 or 48 hours after the immediate delayed retention test when examining the learning effect of a new motor task, this study performed the delayed retention test a week after the immediate delayed retention test to simulate the frequency of outpatient visits in the clinical setting of sports physical therapy. Since there was no significant difference among the three observation methods at the delayed retention test, and intra-group comparisons in each group were observed, it was thought that the practice period used in this study may be insufficient for a newly motor skill task of moderate difficulty to be acquired by a novice, as performed in this study. In addition, it was also thought that the results in the delayed retention test may be influenced by task specificity because it has been reported that the motor learning effect is affected by the task specificity ${ }^{23-25)}$.

In conclusion, this study suggests that MSO was maintained and effective, at least immediately after acquisition of observational learning, but the effect might not be noticeable after 1 week.

The limitation of this study was a possibility that results may be influenced by large inter-individual variability concerning the transformation of ability from motor imagery to actual motor skill. However, since a motor imagery test was not executed in this study, it was thought that further research, which set the motor imagery test and longer periods of acquisition phases in motor learning, will probably be necessary to clarify the differences among observation methods, including MSO, MO and $\mathrm{SO}$, to facilitate the correct modification of sports movement in sports physical therapy.

\section{REFERENCES}

1) Zachazewski JE, Felder CR, Knortz K, et al.: Competency revalidation study: a description of advanced clinical practice in sports physical therapy. J Orthop Sports Phys Ther, 1994, 20: 110-124. [Medline] [CrossRef]

2) Weber MD, Thein-Nissenbaum J, Bartlett L, et al.: Competency revalidation study of specialty practice in sports physical therapy. N Am J Sports Phys Ther, 2009, 4: 110-122. [Medline]

3) Ellenbecker TS, Nirschl R, Renstrom P: Current concepts in examination and treatment of elbow tendon injury. Sports Health, 2013, 5: 186-194. [Medline] [CrossRef]

4) Nirschl RP, Sobel J: Conservative treatment of tennis elbow. Phys Sportsmed, 1981, 9: 43-54. [Medline] [CrossRef]

5) Ollivierre CO, Nirschl RP: Tennis elbow. Current concepts of treatment and rehabilitation. Sports Med, 1996, 22: 133-139. [Medline] [CrossRef]

6) Batt ME: A survey of golf injuries in amateur golfers. Br J Sports Med, 1992, 26: 63-65. [Medline] [CrossRef]

7) Villaseñor-Ovies P, Vargas A, Chiapas-Gasca K, et al.: Clinical anatomy of the elbow and shoulder. Reumatol Clin, 2012, 8: 13-24. [Medline] [CrossRef]

8) Salmoni AW, Schmidt RA, Walter CB: Knowledge of results and motor learning: a review and critical reappraisal. Psychol Bull, 1984, 95: 355-386. [Medline] [CrossRef]

9) Schmidt RA, Young DE, Swinnen S, et al.: Summary knowledge of results for skill acquisition: support for the guidance hypothesis. J Exp Psychol Learn Mem Cogn, 1989, 15: 352-359. [Medline] [CrossRef]

10) Winstein CJ, Schmidt RA: Reduced frequency of knowledge of results enhances motor skill learning. J Exp Psychol Learn Mem Cogn, 1990 , 16: 677-691. [CrossRef]

11) Barzouka K, Bergeles N, Hatziharistos D: Effect of simultaneous model observation and self-modeling of volleyball skill acquisition. Percept Mot Skills, 2007, 104: 32-42. [Medline] [CrossRef]

12) Marques PG, Corrêa UC: The effect of learner's control of self-observation strategies on learning of front crawl. Acta Psychol (Amst), 2016,164 : 151-156. 
[Medline] [CrossRef]

13) Sale P, Ceravolo MG, Franceschini M: Action observation therapy in the subacute phase promotes dexterity recovery in right-hemisphere stroke patients. BioMed Res Int, 2014, 2014: 457538. [Medline] [CrossRef]

14) Park EC, Hwangbo G: The effects of action observation gait training on the static balance and walking ability of stroke patients. J Phys Ther Sci, 2015, 27: 341-344. [Medline] [CrossRef]

15) Keysers C, Gazzola V: Social neuroscience: mirror neurons recorded in humans. Curr Biol, 2010, 20: R353-R354. [Medline] [CrossRef]

16) Lee D, Roh H, Park J, et al.: Drinking behavior training for stroke patients using action observation and practice of upper limb function. J Phys Ther Sci, 2013, 25: 611-614. [Medline] [CrossRef]

17) Magill RA, Schoenfelder-Zohdi B: A visual model and knowledge of performance as a source of information for learning a rhythmic gymnastics skill. Int J Sport Psychol, 1996, 27: 7-22.

18) Dowrick PW: A review of self-modeling and related interventions. Appl Prev Psychol, 1999, 8: 23-39. [CrossRef]

19) Lefebvre S, Dricot L, Laloux P, et al.: Neural substrates underlying stimulation-enhanced motor skill learning after stroke. Brain, 2015, 138: 149-163. [Medline] [CrossRef]

20) Farquharson AL, Cresswell AC, Beard JD, et al.: Randomized trial of the effect of video feedback on the acquisition of surgical skills. Br J Surg, 2013, 100: 1448-1453. [Medline] [CrossRef]

21) Lee TD, Swinnen SP, Serrien DJ: Cognitive effort and motor learning. Quest, 1994, 46: 328-344. [CrossRef]

22) Evers G: Everything golf—golf swing instruction, golf putting instruction: how to play golf, best pro to beginner golf instruction. Great Britain: CreateSpace Independent Publishing Platform, 2003, pp 47-83.

23) Kattner F, Cox CR, Green CS: Transfer in rule-based category learning depends on the training task. PLoS One, 2016, 11: e0165260 [CrossRef]. [Medline]

24) Deveau J, Seitz AR: Applying perceptual learning to achieve practical changes in vision. Front Psychol, 2014, 5: 1166. [Medline] [CrossRef]

25) Green CS, Bavelier D: Learning, attentional control, and action video games. Curr Biol, 2012, 22: R197-R206. [Medline] [CrossRef] 